\title{
A Novel Method of Antibacterial Evaluation Based on the Inhibition of Hydrogen Sulfide Producing Activities of Salmonella -Using Copper as a Model Antibacterial Agent-*
}

\author{
Yutaka Midorikawa ${ }^{1}$, Masaaki Nakai ${ }^{2}$, Kaoru Midorikawa $^{3}$ and Mitsuo Niinomi ${ }^{2}$ \\ ${ }^{1}$ Faculty of Health, Suzuka University of Medical Science, Suzuka 510-0293, Japan \\ ${ }^{2}$ Institute for Materials Research, Tohoku University, Sendai 980-8577, Japan \\ ${ }^{3}$ Graduate School of Medicine, Mie University, Tsu 514-8507, Japan
}

\begin{abstract}
A novel method for detecting antimicrobial activity using an innate property of the Salmonella bacteria, namely, the ability of Salmonella to produce hydrogen sulfide $\left(\mathrm{H}_{2} \mathrm{~S}\right)$ was developed in this study. The validity of the method was evaluated by comparing the antibacterial activity of copper to that of aluminum. Salmonella was inoculated over the entire surface of deoxycholate hydrogen sulfide lactose (DHL) agar plates that included Ammonium ferric citrate $\left(\mathrm{C}_{6} \mathrm{H}_{8} \mathrm{FeN}\right)$. Approximately $25 \mu \mathrm{L}$ of cupric chloride $\left(\mathrm{CuCl}_{2}, 1 \%\right.$ weight ratio) solution or aluminum chloride $\left(\mathrm{AlCl}_{3}, 1 \%\right.$ weight ratio) solution was added to the center of the medium. The surface of the medium was covered with polyethylene terephthalate (PET) films to induce an anaerobic state. Salmonella was cultured under anaerobic conditions at $310 \mathrm{~K}\left(37^{\circ} \mathrm{C}\right)$ for $86.4 \mathrm{ksec}(24 \mathrm{~h})$. The antibacterial activity of copper was determined by observing the medium surface color change due to iron sulfide (FeS) formation, which was caused by the production of $\mathrm{H}_{2} \mathrm{~S}$ by Salmonella; blackness indicated presence of newly formed FeS. A quantitative evaluation of copper's antimicrobial activity was performed using a gradient of $\mathrm{CuCl}_{2}$ concentrations; results were compared with those of the present standard method, Kirby-Bauer disk diffusion method on the Mueller Hinton medium. Finally, in order to evaluate the antibacterial activity of metals, Salmonella was inoculated on DHL agar plates. Subsequently, Japanese coins (1-yen, 5-yen, 10-yen, 50-yen, 100-yen, and 500-yen coins) were placed on the agar and cultured at $310 \mathrm{~K}$ for $86 \mathrm{ksec}$. Salmonella cultured in the presence of $\mathrm{AlCl}_{3}$ produces black color, while no blackening is observed with $\mathrm{CuCl}_{2}$, suggesting that copper possesses an antibacterial property against Salmonella. $\mathrm{CuCl}_{2}$ suppresses $\mathrm{H}_{2} \mathrm{~S}$ production by Salmonella, as copper ions form a transparent circle or ellipse (new halo) around the point at which $\mathrm{CuCl}_{2}$ had has been plated. The size of the new halo increases in direct proportion to the concentration of $\mathrm{CuCl}_{2}$. The halo is no longer visible at $0.034 \mathrm{mg}$ of $\mathrm{CuCl}_{2}$ in our method, while the halo disappears with $4.34 \mathrm{mg}$ of $\mathrm{CuCl}_{2}$ in the Kirby-Bauer test. Therefore, the present method is 129 times more sensitive than the standard method, suggesting increased usefulness and effectiveness in testing antibacterial activity. No FeS-dependent black circle is formed under any of the coins, with the exception of the 1-yen coin, which contains aluminum and no copper. Therefore, the copper-containing coins have an antibacterial effect. [doi:10.2320/matertrans.M2016007]
\end{abstract}

(Received January 15, 2016; Accepted March 10, 2016; Published April 8, 2016)

Keywords: salmonella, copper, antimicrobial, hydrogen sulfide, iron sulfid

\section{Introduction}

Among metals with antibacterial activities, silver and copper have long been known for their toxicity towards microorganisms but relative safety to the human body. On the other hand, titanium, which is recognized as a promising medical material for implants and other purposes, and most familiar metals including iron and aluminum, do not have antibacterial properties in themselves ${ }^{1-3)}$. The antibacterial activities of metals are not generally expressed by themselves but by the metallic ions derived from them.

Today, a wide range of antibacterial products is available in the market, including kitchen accessories to enhance food hygiene, clothing such as underwear and stockings, and bath/ toiletry goods. The method to evaluate the antibacterial activity and efficacy on the surface of these plastic/metal/ceramic products with antibacterial finishing is defined by the Japan Industrial Standards (JIS) Z 2801: 20104). The specified method for evaluation of the antibacterial activities of a material involves the following steps: bringing the subject in contact with a solution containing the gram negative Escherichia coli (E. coli) or the gram positive Staphylococcus aureus, culturing the mixture on agar medium available in market at $310 \mathrm{~K}$ (37 degrees C) for $86.4 \mathrm{ks}(24 \mathrm{~h})$, counting the number

*This Paper was Originally Published in Japanese in J. Japan Inst. Met. Mater. 80 (2016) 165-170. The title of the paper is changed. of viable bacteria contained in $1 \mathrm{~mL}$ of the solution, and comparing the result with the bacterial count of the control group that was not allowed the bacterial solution to contact with the subject. However, this method is cost- and labor-intensive due to the requirements of solution preparation and colony counting. Therefore, the authors designed an alternative method exploiting the hydrogen sulfide $\left(\mathrm{H}_{2} \mathrm{~S}\right)$-producing property of Salmonella bacteria.

The Salmonella bacterium, one of the causative agents of food poisoning, is characterized by its significant production of $\mathrm{H}_{2} \mathrm{~S}$ during growth. $\mathrm{H}_{2} \mathrm{~S}$ produced by Salmonella reacts with iron $(\mathrm{Fe})$, if present in the culture medium, to form black colored iron sulfide (FeS). Therefore, agar medium containing $\mathrm{Fe}$ in the form of iron citrate shows color change upon $\mathrm{H}_{2} \mathrm{~S}$ production by Salmonella, thus allowing visual evaluation of antibacterial activity. FeS is formed in mildly acidic conditions and is dissolved in strong acid/alkali ${ }^{5}$. These characteristics of Salmonella allow antibacterial evaluation simply by verifying the formation of black FeS, and are therefore expected to decrease the cost and labor involved in determining antibacterial activities. The simple procedure of culturing $\mathrm{H}_{2} \mathrm{~S}$-producing bacteria including Salmonella will eliminate the necessity of counting viable bacteria for statistical comparison with the control group.

Therefore, this study evaluates the antibacterial activities of copper ions with this novel antibacterial evaluation method to investigate its potential. The current method used for test- 
ing antibacterial sensitivity, or the Kirby-Bauer test ${ }^{6)}$ was used as the control method instead of the method specified by JIS (JIS Z $2801: 2010^{4)}$ ) because the latter excludes the use of strains other than E. coli and Staphylococcus aureus. However, the Kirby-Bauer test imposes no restraint on the strains to be uses, allowing the use of Salmonella for direct comparison of test results with the same subject microorganism.

\section{Experimental Procedures}

\subsection{Bacterial strain and culture medium}

Non-typhi Salmonella enterica subsp. enterica serovar Virchow ${ }^{7)}$ that produces a large amount of $\mathrm{H}_{2} \mathrm{~S}$ was used as a bacteria strain in this study.

Deoxycholate-hydrogen sulfide-lactose (DHL) medium (Eiken Chemical, Japan) was used as the culture medium. This medium does not require any sterilization and is characterized by the presence of iron citrate $\left(\mathrm{C}_{6} \mathrm{H}_{5} \mathrm{FeO}_{7}\right)$ as the iron source, and sodium thiosulfate $\left(\mathrm{Na}_{2} \mathrm{O}_{3} \mathrm{~S}_{2}\right)$ as the source of sulfur. In this medium, the metabolism of Salmonella results in the release of sulfur from sodium thiosulfate and production of $\mathrm{H}_{2} \mathrm{~S}$, which reacts with $\mathrm{Fe}$ contained in iron citrate to form the black-colored FeS. The resulting color change to black indicates the detection of the strain. For preparation of DHL media, $63 \mathrm{~g}$ of powdered medium was dissolved in $1,000 \mathrm{~mL}$ of distilled water, maintained at $373 \mathrm{~K}$, and subsequently $20 \mathrm{~mL}$ of the solution was dispensed in an 80-mm petri dish, and allowed to solidify at room temperature. Multiple dishes were prepared. For the control Kirby-Bauer test ${ }^{8)}$, $38 \mathrm{~g}$ of powdered Mueller-Hinton medium (Eiken Chemical) was dissolved in $500 \mathrm{~mL}$ of distilled water. The solution was sterilized at high pressure and high temperature for $1.2 \mathrm{ksec}$ at $394 \mathrm{~K}$, and $20 \mathrm{~mL}$ of the solution was dispensed in a petri dish, and solidified at room temperature. Multiple dishes were prepared.

\subsection{Subject metals to evaluate antibacterial activity}

Solution of copper and aluminum ions for qualitative and quantitative evaluation of antibacterial activity were used in this study. The detailed procedures are as follows.

Qualitative testing: $1 \mathrm{~g}$ of copper (II) chloride dihydrate $\left(\mathrm{CuCl}_{2} \cdot 2 \mathrm{H}_{2} \mathrm{O}\right)$, which is a copper ion compound with antibacterial activities, was dissolved in $100 \mathrm{~mL}$ of distilled water to prepare a $1 \%$ solution $(0.06 \mathrm{~mol})$. As a control, $1 \mathrm{~g}$ of aluminum chloride $\left(\mathrm{AlCl}_{3} \cdot 6 \mathrm{H}_{2} \mathrm{O}\right)$, which has no antibacterial property, was dissolved in $100 \mathrm{~mL}$ of distilled water (0.04 mol).

Quantitative testing: $7 \mathrm{~g}$ of $\mathrm{CuCl}_{2} \cdot 2 \mathrm{H}_{2} \mathrm{O}$ was dissolved in $10 \mathrm{~mL}$ of distilled water $(4.1 \mathrm{~mol}$ :to form a nearly saturated solution), and was then subjected to two-fold serial dilution to prepare solutions of $0.6836,1.367,2.734,5.469,10.94$, $21.88,43.75,87.5,175,350$, and $700 \mathrm{mg} / \mathrm{mL}$.

\subsection{Culture method under anaerobic conditions}

Anaerobic conditions were induced using a transparent polyethylene terephthalate (PET) film that was sterilized at high temperature $394 \mathrm{~K}$ under high pressure for $1.2 \mathrm{ks}$. The $0.2-\mathrm{mm}$ thick PET film was cut into squares of $20 \mathrm{~mm}$ and $50 \mathrm{~mm}$, which were placed in close contact with the medium surface to block the air and establish an anaerobic state.

\subsection{Procedure for the quantitative and qualitative evalu- ation of antibacterial activities}

The procedure comprises the following four steps.

(1) Preparation of Salmonella culture: The strain was inoculated on a DHL agar plate with a nichrome loop, and incubated at $310 \mathrm{~K}$ for $86.4 \mathrm{ks}$ to obtain a discrete colony of Salmonella. The colony was picked with a cotton swab tip and applied to the entire surface of the DHL agar medium in a fresh petri dish.

(2) Qualitative evaluation: A 0.2-mm thick 20-mm square transparent PET film was immersed in $1 \% \mathrm{CuCl}_{2}$ solution and $1 \% \mathrm{AlCl}_{3}$ solution, respectively to evaluate the difference in antibacterial activities between copper ions and aluminum ions. The film was dried and placed in close contact with the DHL agar medium to generate an anaerobic condition and the culture was incubated at $310 \mathrm{~K}$ for $86.4 \mathrm{ks}$. As a control, a 20 -mm square transparent film with no treatment was incubated in the same manner as the test culture.

(3) Quantitative evaluation: To the center of the DHL agar medium, $25 \mu \mathrm{L}$ of $\mathrm{CuCl}_{2}$ solutions of concentrations ranging from $0.6836 \mathrm{mg} / \mathrm{mL}$ to $700.0 \mathrm{mg} / \mathrm{mL}$ were dropped. The spot was then covered with a $0.2 \mathrm{~mm}$-thick $50-\mathrm{mm}$ square PET film to generate an anaerobic condition and was then incubated at $310 \mathrm{~K}$ for $86.4-\mathrm{ks}$.

The control Kirby-Bauer test was performed as follows:

As indicated above, $25 \mu \mathrm{L}$ each of $\mathrm{CuCl}_{2}$ solutions of varied concentrations were dropped on a $10-\mathrm{mm}$ paper disk (Blaine test paper, ADVANTEC, Japan). The disk, infiltrated with $\mathrm{CuCl}_{2}$ solution, was then placed on the Mueller-Hinton agar plate entirely inoculated with Salmonella and was incubated at $310 \mathrm{~K}$ for $86.4 \mathrm{ks}$. The PET film was not used because the Kirby-Bauer test does not require anaerobic conditions on the medium surface.

(4) Evaluation of antibacterial activities

The DHL agar media inoculated with Salmonella showed blackening due to $\mathrm{FeS}$ formation in the anaerobic region, but no change in the region where the antibacterial metal ion solution was added because $\mathrm{H}_{2} \mathrm{~S}$ was not produced in that area. The area that showed no blackening was circular or elliptical shape because the metal ion solution had spread radially. The surface area of this circular or elliptical shape was measured as an index of the strength of the antibacterial effect. On the other hand, in the control Kirby-Bauer test using the Mueller-Hinton agar, a round zone of inhibition was formed around the disk after incubation where no bacterial growth was observed. The diameter of this inhibition was also measured (according to the current standard method).

\subsection{Application to an antibacterial test of metals}

The Japanese 1-yen coin of aluminum, 5-yen coin of copper-zinc alloy, 10-yen coin of copper-tin alloy, and $50 / 100 / 500$-yen coins of nickel-copper alloy were placed on the DHL agar media entirely inoculated with Salmonella. A transparent $0.2-\mathrm{mm}$ thick $20-\mathrm{mm}$ square PET film was used as the control. The coins were incubated on the medium at $310 \mathrm{~K}$ for $86.4 \mathrm{ks}$, and were then then removed to compare the change in the medium color underneath. 


\section{Results and Discussion}

\subsection{Effects of anaerobic conditions on the formation of FeS by Salmonella}

Figure 1 shows a plate containing the DHL agar medium entirely inoculated with Salmonella and incubated under a PET film at $310 \mathrm{~K}$ for $86.4 \mathrm{ks}$. Growth of Salmonella is detected by blackening of the medium under anaerobic conditions generated by the PET film. A typical characteristic of non-typhi Salmonella is the abundant production of $\mathrm{H}_{2} \mathrm{~S}$ using sulfur sources such as thiosulfuric acid present in the DHL medium. Active growth of Salmonella is therefore confirmed by blackening of the medium due to FeS formed by the reaction between $\mathrm{H}_{2} \mathrm{~S}$ produced by Salmonella and iron (III) citrate included in the medium. Since the PET film itself is not antibacterial in nature, active growth of Salmonella is observed underneath it. However, the area that is not covered with the PET films does not exhibit any blackening. Probably because the $\mathrm{FeS}$ generated is oxidized again in the aerobic condition. Exposure to oxygen supply in the air resulted in loss of black color. On the other hand, FeS generated from $\mathrm{H}_{2} \mathrm{~S}$ does not seem to be re-oxidized in the anaerobic condition where oxygen supply from the air is blocked by the PET film.

\subsection{Antibacterial activities of copper ions and aluminum ions}

Figure 2 shows a plate containing the DHL agar medium entirely inoculated with Salmonella and incubated under a PET film at $310 \mathrm{~K}$ for $84.6 \mathrm{ks}$. The PET film impregnated with copper ions or aluminum ions was placed on the medium surface to induce an anaerobic condition. Blackening is confirmed under the PET film treated with aluminum ions whereas no blackening is observed under that treated with copper ions. These color reactions indicate that $\mathrm{FeS}$ is generated in the presence of aluminum ions that do not inhibit $\mathrm{H}_{2} \mathrm{~S}$ pro-

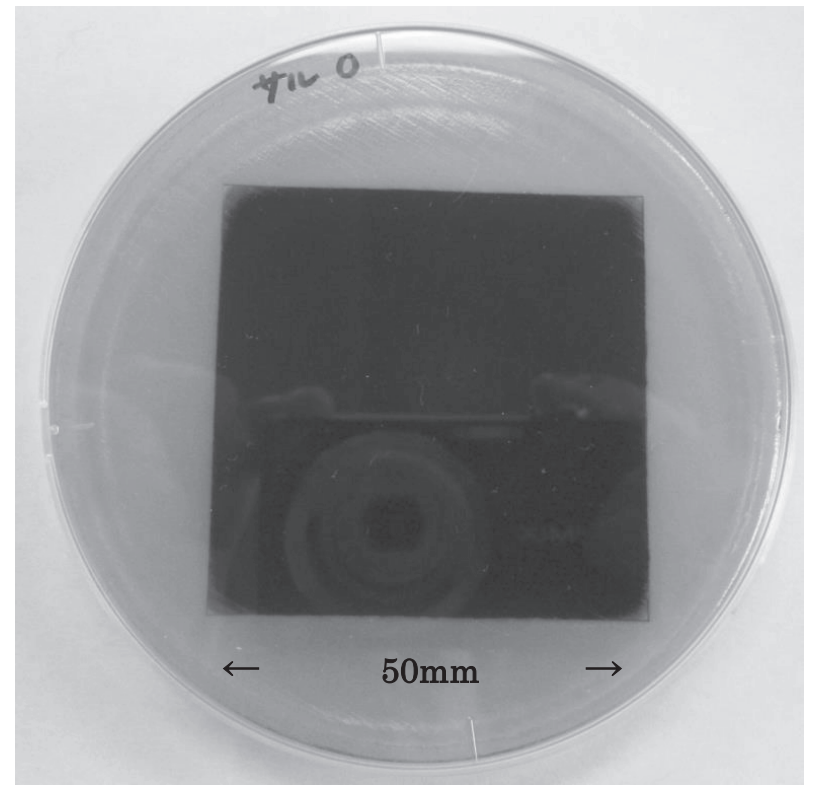

Fig. 1 Iron sulfide formation by the reaction between hydrogen sulfide produced by Salmonella and iron (III) citrate in the medium under anaerobic conditions. duction by Salmonella whereas it is not generated in the presence of copper ions due to inhibition of $\mathrm{H}_{2} \mathrm{~S}$ production. This indicates that copper ions have antibacterial effects whereas aluminum ions do not, which is a known fact ${ }^{1-3)}$. Therefore, this study demonstrated for the first time that antibacterial activity can be determined by the formation of FeS due to the $\mathrm{H}_{2} \mathrm{~S}$ produced by Salmonella.

\subsection{Comparison of antibacterial activity of copper ions as quantified by the novel method and the current standard method}

Figure 3 shows the results of antibacterial activity testing by our proposed method (upper row) and by the current standard Kirby-Bauer method (lower row). In the Kirby-Bauer method, the inhibition zone representing zero bacterial growth appears at a copper ion concentration $\geq 4.37 \mathrm{mg}$. On the other hand, in the novel method, the inhibition zone is shown as a circle or an ellipse appears at a copper ion concentration $\geq 0.0034 \mathrm{mg}$. We have designated the region under the PET film without the black FeS formation as "new halo" to indicate inhibition by the novel method, and the zone of inhibition obtained by the standard method as "old halo." The size of the new halo was found to form in proportion to copper ion concentration. The surface under the PET film outside the new halo exhibits blackening and indicates FeS formation whereas the surface outside the PET film does not show any change in color.

The threshold for the new halo formation was $0.034 \mathrm{mg}$, which is far lower than $4.37 \mathrm{mg}$ that is obtained with the conventional method, and is equivalent to a ratio of approximately $1 / 129$. Thus, the novel method quantitatively displayed higher sensitivity for evaluation of antibacterial activity than the current method did. In addition, when a halo is formed in the standard method, the microorganisms under the paper disk are dead and their growth cannot be visually determined. In contrast, the new halo has a distinct black-white contrast that allows clearer visual evaluation of bacterial growth. Fig-

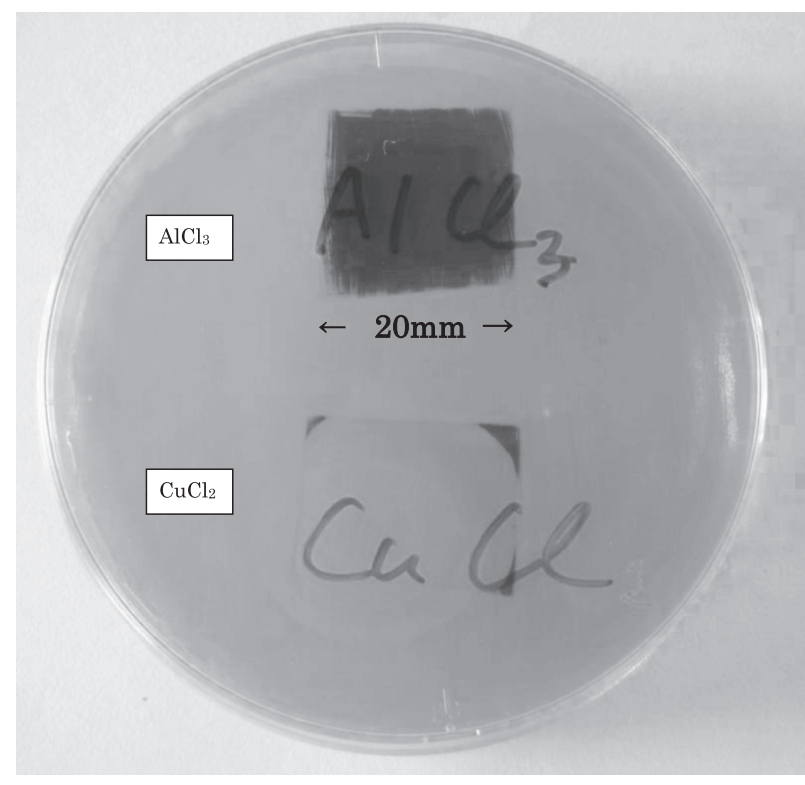

Fig. 2 Effects of copper ions and aluminum ions on FeS formation by Salmonella. 


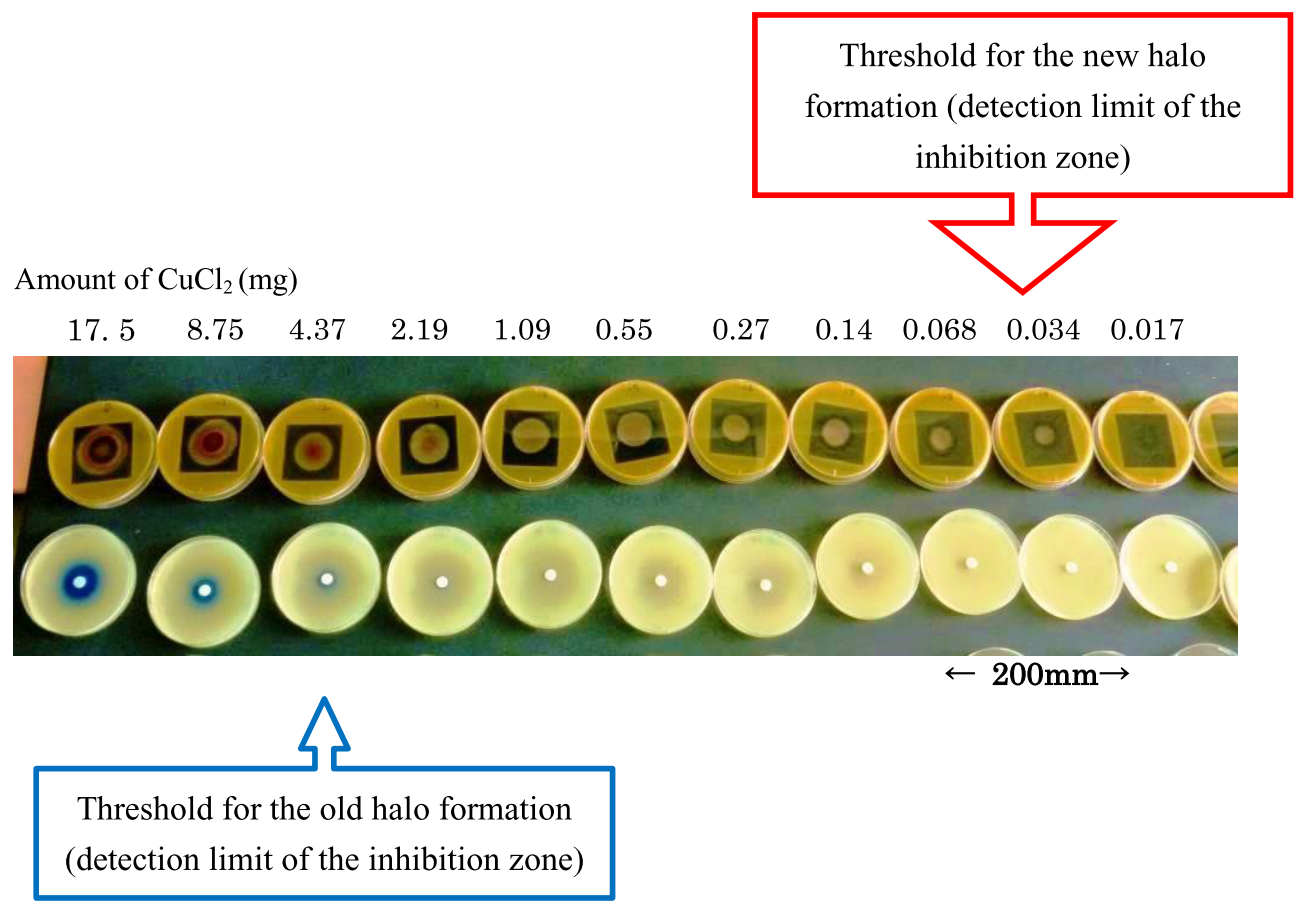

Fig. 3 Comparison of sensitivity between the new method and the Kirby-Bauer method under $\mathrm{CuCl}_{2}$ treatment. Upper: New method using the DHL medium. Lower: Kirby-Bauer method using the Mueller Hinton medium.

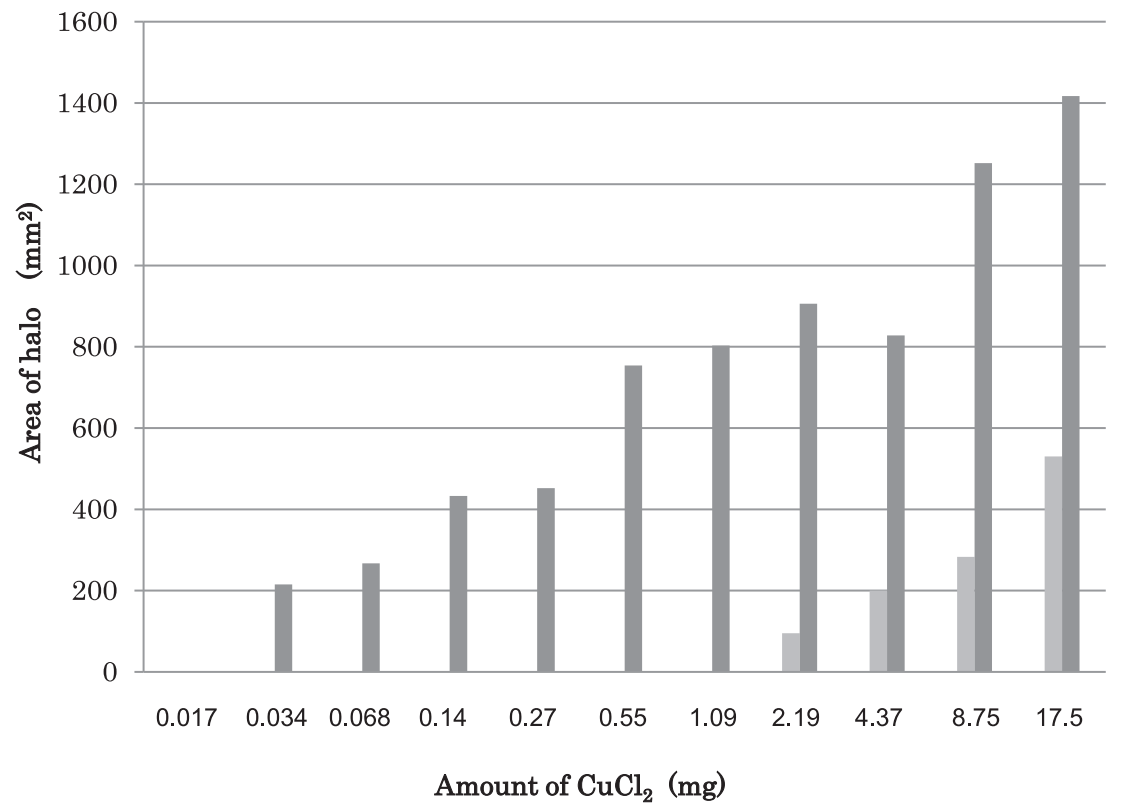

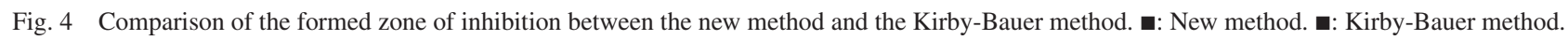

ure 4 shows a graph comparing the surface area in $\mathrm{mm}^{2}$ between the new and old halos with the varied copper ion concentrations shown in Fig. 3. The size of the halo size constitutes another difference between the new and current standard method, with the new halo having a surface area that is 3 to 10 times larger than that of an old halo.

\subsection{Superiority of the method proposed in this study}

Our previous study demonstrated that $\mathrm{H}_{2} \mathrm{~S}$ production is hampered when saline concentration in the medium is increased despite continued growth of Salmonella ${ }^{9)}$. Similarly, the results of this study simply imply that the copper ions may decrease the $\mathrm{H}_{2} \mathrm{~S}$ productivity of Salmonella but that they may not necessarily stop the bacterial growth. Therefore, a decrease in $\mathrm{FeS}$ formation related to $\mathrm{H}_{2} \mathrm{~S}$ production may reflect a lowered rate of multiplication in Salmonella.

The current standard method uses ordinary agar or Mueller-Hinton agar that contains no sulfur or iron source that enables $\mathrm{H}_{2} \mathrm{~S}$ production or FeS formation by the microorganisms. Therefore, determination of growth inhibition in microorganisms requires visual evaluation of the old halo. The minimum concentration of antibacterial materials at 


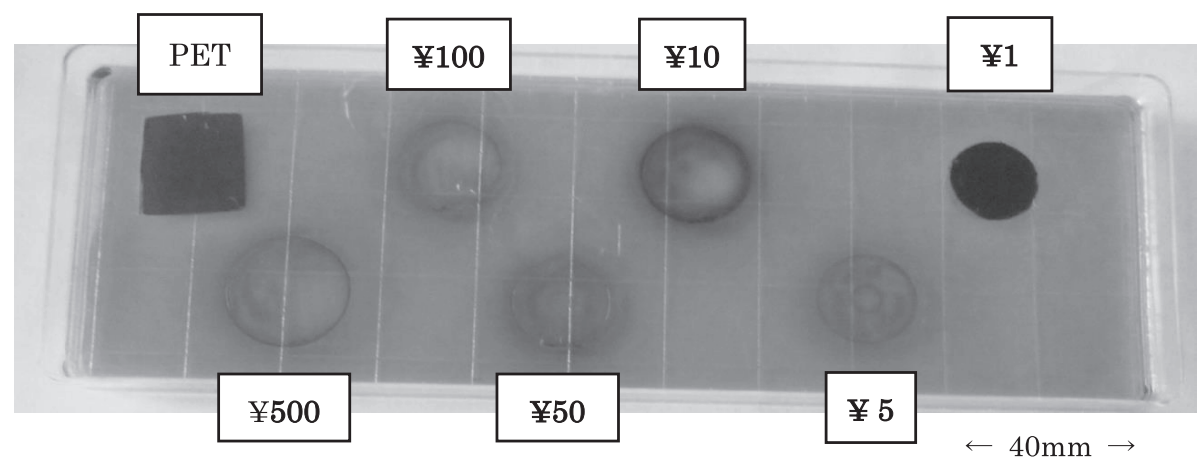

Fig. 5 Effects of coins on Salmonella growth. (¥1: 1-yen coin, ¥:5-yen coin, ¥10: 10-yen coin, ¥50: 50-yen coin, ¥100: 100-yen coin, ¥500: 500-yen coin, PET: Plastic film)

which the formation of the old halo can be visually recognized is called the minimum inhibitory concentration (MIC) which, in this study, was proved significantly higher than the minimum concentration required blocking $\mathrm{H}_{2} \mathrm{~S}$ production in the novel method.

These results imply that the novel method has a higher sensitivity than the current standard method and enables distinct determination of microorganism growth inhibition effects.

\subsection{Application to antibacterial activity testing using coins}

Figure 5 shows the results of antibacterial activity evaluation by the new method. The coins were kept in close contact with the agar such that no air could intrude between the coins and the agar. The blackening underneath the coins due to FeS formation was demonstrated only for the 1-yen coin and the control PET film sheet. Color changes for other coins that were similar to blackening were due to rust. These results indicate the antibacterial effects of copper, which is present in all the coins other than the 1-yen coin. The 1-yen coin is made of aluminum and, therefore, did not exhibit the antibacterial effect seen in the other coins. This simple method of application clearly demonstrates antibacterial activity of copper and is expected to lower the cost and labor required for antibacterial testing. The existing DHL agar was used in this study, but in order to improve the sensitivity further, media with better sensitivity can be developed specifically for the visual evaluation of FeS formed by Salmonella. Further, the copper ion used in this study resists quality degradation (titer decrease) better than organic antibacterial agents including penicillin and other antibiotics. The toxicity of copper in humans that was discussed in the past has now been denied ${ }^{10)}$, thus proving the suitability of copper for a wide range of applications from the perspective of safety. This new evaluation method can be applied to the testing of a wide range of antibacterial metals and materials other than copper. The higher sensitivity of this novel method may serve the discovery of antibacterial substances that may have been overlooked by use of the conventional method.

This novel method can be used to test a wide range of products and reagents for antibacterial properties, including dishes, cleaners, and antibiotics. We will continue to investigate these possibilities in the future.

\section{Conclusions}

A new method of antibacterial activity evaluation by exploiting formation of $\mathrm{FeS}$ due to $\mathrm{H}_{2} \mathrm{~S}$ produced by Salmonella was developed and proved the effectiveness of the novel method using copper, which has known antibacterial properties in this study. In addition, the results of the novel method were compared with those of the current standard method Kirby-Bauer test. The following results were obtained.

(1) Salmonella was inoculated entirely over the DHL agar medium containing sources of sulfur and iron. A PET film sheet was placed over part of the medium to induce an anaerobic condition and the dish was incubated at $310 \mathrm{~K}$ for $86.4 \mathrm{ks}$. The anaerobic part of the medium was blackened with the $\mathrm{FeS}$ formed due to the reaction between $\mathrm{H}_{2} \mathrm{~S}$ produced by Salmonella and Fe contained in the DHL agar.

(2) Using a copper as an antibacterial substance and aluminum as the control substance, the part of medium covered with the PET film containing aluminum ions was blackened, whereas that with the PET film containing copper ions shows no blackening. Thus, the antibacterial property of copper was visually determined.

(3) In the test that used copper ion solutions of differing concentrations, the zone of inhibition was formed with its center at the point where the copper solution was dropped and the area of the circle was proportional to the copper ion concentration. A comparison of the novel method with the standard Kirby-Bauer test suggested that the former method had a superior sensitivity equivalent to 129 times that of the current standard method. The surface area of inhibition is also 3 to 10 times larger with the novel method than that of the method.

(4) Different coins that are in circulation in Japan were placed each other on the DHL agar medium entirely inoculated with Salmonella. The blackening of medium due to FeS formation was observed only under the 1-yen coin made of aluminum. All other coins contain copper, and therefore showed antibacterial activities.

(5) This novel and simple method for highly sensitive evaluation of antibacterial activity was developed based on FeS formation using Salmonella and was proposed for the first time in this study. Further improvement of the method or development of other methods that apply this principle can be expected to improve the productivity and performance of the antibacterial evaluation. 


\section{Acknowledgment}

This study was a joint research effort with the Institute for Materials Research, Tohoku University (Grant number $14 \mathrm{~K} 0005,15 \mathrm{~K} 0018$ ) and was funded by JSPS Kaken with Grant number 15K00894 to which we sincerely express our gratitude.

The manuscript was edited and proofread by a translation agency, a priori, Inc. Tokyo, Japan.

\section{REFERENCES}

1) H.E. Müller: Zentralbl. Bakteriol. Mikrobiol. Hyg. [B] 182 (1985) 95101.

2) R. Shrestha, D. R. Joshi, J. Gopali and Sujan Piya: Nepal J. Science and
Technology. 10 (2009) 189-193.

3) C. Dhabuwala: J. Sex. Med. 7 (2010) 3516-3519.

4) Antimicrobial products-Test for antimicrobial activity and efficacy. JIS Z 2801 (2000)

5) L. Stoffels, Martin Krehenbrink, B. C. Berks and G. Unden: J. Bac. 194 (2012) 475-485.

6) A.W. Bauer, D.M. Perry and W.M.M. Kirby: Arch. Intern. Med. 104 (1959) 208-216.

7) M. Weinberger, H. Solnik-Isaacb, D. Shacharb, A. Reisfeldc, L. Valin skyc, N. Andornc, V. Agmonc, R. Yishaic, R. Bassald, A. Frasere, S. Yaronb and D. Cohend: Clin. Microbiol. Infect. 12 (2006) 999-1005.

8) W.L. Drew, A.L. Barry, R.O. Toole and J.C. Sherris: Appl. Microbiol. 24 (1972) 240-247.

9) Y. Midorikawa, S. Nakamura, R. Phetsouvanh and K. Midorikawa: Open J. Med. Microbiol. 04 (2014) 90-95.

10) S.S. Percival and E.D. Harris: Am. J. Physiol. Cell Physiol. 258 (1990) 140-146. 